\title{
USO DO PNEUMOPERITÔNIO NO PRE-OPERATÓRIO DE HERNIA INCISIONAL GIGANTE: UM RELATO DE CASO
}

\author{
Alfredo LIMA FILHO ${ }^{1}$ \\ Camila Carvalho CAVALCANTE ${ }^{2}$ \\ Diogo Ramalho Tavares MARINHO ${ }^{3}$ \\ Paulo Vinicius Alves LOPES ${ }^{4}$ \\ Rafael Calvão BARBUTO ${ }^{5}$ \\ Renan Farias Rolim VIANA ${ }^{6}$ \\ Ticiana de Magalhães Benevides LIMA ${ }^{7}$
}

\author{
1 - Residente de Cirurgia Geral do Hospital das Clínicas da UFMG \\ (e-mail: alfredofilho@hotmail.com) \\ 2 - Residente de Cirurgia Geral do Hospital Luxemburgo \\ (email: camila_ccavalcante@hotmail.com) \\ 3 - Residente de Cirurgia Geral do Hospital das Clínicas da UFMG \\ (e-mail: drtm_7@hotmail.com) \\ 4 - Residente de Cirurgia Geral do Hospital das Clínicas da UFMG \\ (e-mail: paulovinicius.lopes@gmail.com) \\ 5 - Preceptor e Chefe da Residência de Cirurgia Geral do Hospital das Clínicas da UFMG \\ (email: rafaelbarbuto@gmail.com) \\ 6 - Residente de Cirurgia Geral do Hospital das Clínicas da UFMG \\ (e-mail:renanfrv@gmail.com) \\ 7 - Médica da Força Aérea Brasileira \\ (email: ticianabenevides@ gmail.com)
}

Recebido em: 09/04/2015 - Aprovado em: 06/07/2015 - Disponibilizado em: 15/07/2015

Resumo: A Hérnia incisional gigante com perda de domicílio possui grandes limites técnicos ao cirurgiões devido ao potencial de complicações relacionados ao seu reparo, como a temida síndrome compartimental abdominal, caracterizada pela hipertensão abdominal associada a redução do retorno venoso, débito cardíaco, diurese e piora do padrão ventilatório sendo potencialmente fatal. Para evitar que a mesma ocorra, foi descrita inicialmente por GoñiMoreno a técnica do pneumoperitônio pré-operatório no tratamento deste tipo de hérnia. O presente relato de caso consiste na utilização desta técnica no reparo de uma hérnia ventral gigante com perda de domicílio, o qual obteve-se sucesso cirúrgico satisfatório, sem a ocorrência de complicações em pós operatório imediato ou recidivas a longo prazo. O trabalho demonstra a efetividade do pneumoperitônio pré-operatório em aumentar o continente para adequar-se ao conteúdo das hérnias incisionais gigantes com perda de domicílio e redução da incidência da síndrome compartimental. Consideramos então a técnica efetiva e segura e sugerimos mais publicações de trabalho acerca da mesma para padronização da técnica.

Palavras-chave: Pneumoperitônio. Hérnia Incisional. Perda de Domicílio. Síndrome Compartimental. Hernioplastia. 


\begin{abstract}
Massive incisional hernia with loss of abdominal domain is a surgical challenge due to a high potential of complications related to this repair witch the main problem is abdominal compartmental syndrome caractherized by abdominal hypertension associated to low venous return, cardiac debit, diuresis and the decrease of respiratory standard that could be lethal. To avoid this complication Goñi-Moreno describe a technique that uses preoperative progressive pneumoperitoneum to treat patients with abdominal-wall hernias. In this related case we have treated this giant ventral hernia with loss of abdominal domain with pneumoperitoneum which was a successful surgical procedure without any early or late complication. This study evaluates the efficiency of preoperative progressive pneumoperitoneum to permit the abdominal cavity to get adapted to the giant incisional hernia with loss of domain and reduct the incidence of compartimental syndrome. We conclude that the surgical technique of pneumoperitoneum is safe and effective and we suggest to increase the studies to approach this surgical technique.
\end{abstract}

Key Words: Pneumoperitoneum; Ventral Hernias; Abdominal Compartimental Syndrome; Hernioplasty.

\section{INTRODUÇÃO}

O reparo das hérnias ventrais abdominais constituem um grande desafio para os cirurgiões devido a dificuldade técnica e altos índices de complicações locais e sistêmicas, principalmente respiratórias e cardiovasculares. Nas hérnias com perda de domicílio, há uma diminuição da pressão intracavitária que, consequentemente, produz queda do retorno venoso, vasodilatação e estase venosa abdominal, assim como redução da capacidade pulmonar, por enfraquecimento do diafragma ao não encontrar resistência durante sua contração.

A técnica de induzir o pneumoperitônio progressivo pré-operatório permite um aumento da pressão intrabdominal de forma gradual, estabiliza a forma e função diafragmática, melhorando a função respiratória, e distende a parede abdominal, permitindo o aumento do volume da cavidade abdominal. Portanto, é recomendada naquelas hérnias ventrais na qual há uma grande quantidade de vísceras no saco herniário visando a redução da incidência de síndrome compartimental abdominal (PINGREE, 1968; MASON, 2001; OLIVEIRA, 2003; MINOSSI, 2009).

O presente trabalho visa relatar um caso de hérnia incisional ventral com perda de domicílio e seu tratamento utilizando técnica de pneumoperitônio preoperatório.

\section{DESCRIÇÃO DO CASO}

Paciente L.G, 59 anos, admitido no ambulatório do grupo de Parede Abdominal e Retroperitônio do Hospital das Clinicas-UFMG (HCUFMG) com queixa de abaulamento em cicatriz de laparotomia mediana xifopúbica. Referia ter sido submetido a cirurgia de urgência devido a abdome agudo perfurativo por corpo estranho (espinha de peixe) em 2012. 
FIGURA 1- Punção de cateter guiado por ultrassom
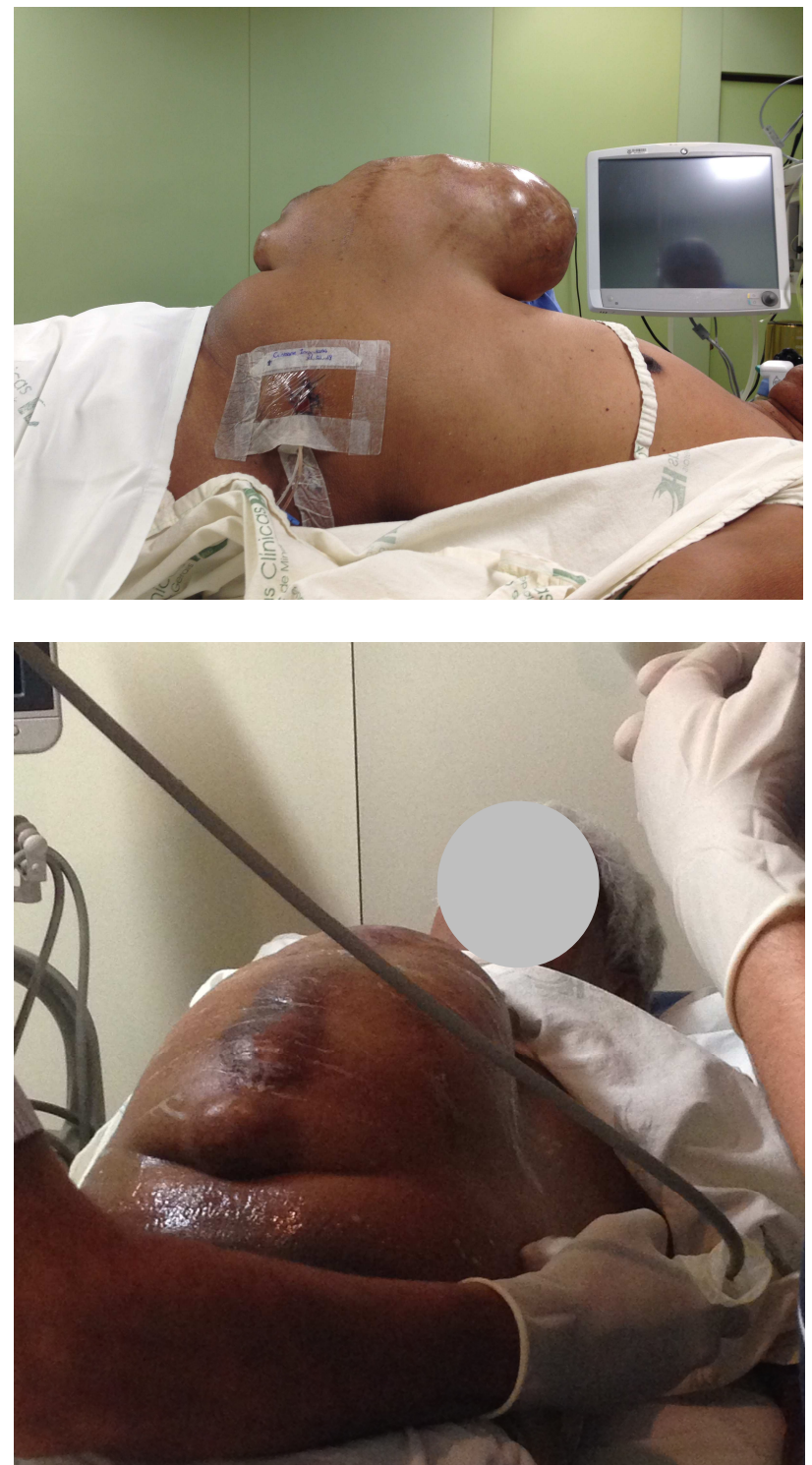

Fonte: Fotos tiradas no próprio serviço.

Relatava que o abaulamento foi inicialmente notado, em menores proporções na primeira semana de pós operatório, principalmente sob manobra de Valsalva, mas houve crescimento gradual e continuo, que tomou grandes proporções e o motivou a procurar atendimento a um grupo especializado. Negava alterações do hábito intestinal, bem como miccional. Negava tosse crônica e quaisquer outros sintomas causadores de aumento da pressão intrabdominal.

Ao exame direcionado, paciente apresentava volumosa hérnia incisional, com anel herniário de cerca de $8 \mathrm{~cm}$, no seu maior diâmetro, decorrente da laparotomia supracitada, com impossibilidade de redução manual do conteúdo herniado, embora não houvesse sinal de encarceramento, o que caracterizava uma hérnia com perda de domicílio.

Paciente foi internado na enfermaria cirúrgica no dia 19/03/14 para confecção de pneumoperitônio preoperatório. Puncionado, sob técnica de Seldinger guiado por ultrassom, um cateter duplo lúmen de acesso venoso central em fossa ilíaca esquerda na cavidade abdominal. Foi insuflado, com auxilio de dispositivo tri-way e seringa de $60 \mathrm{~mL}$, um total de $6420 \mathrm{~mL}$ de ar ambiente, sendo, em média, por sessão diária, insuflado $802,5 \mathrm{~mL}$

FIGURA 2 - Resultado final do pneumoperitônio no pré operatório imediato

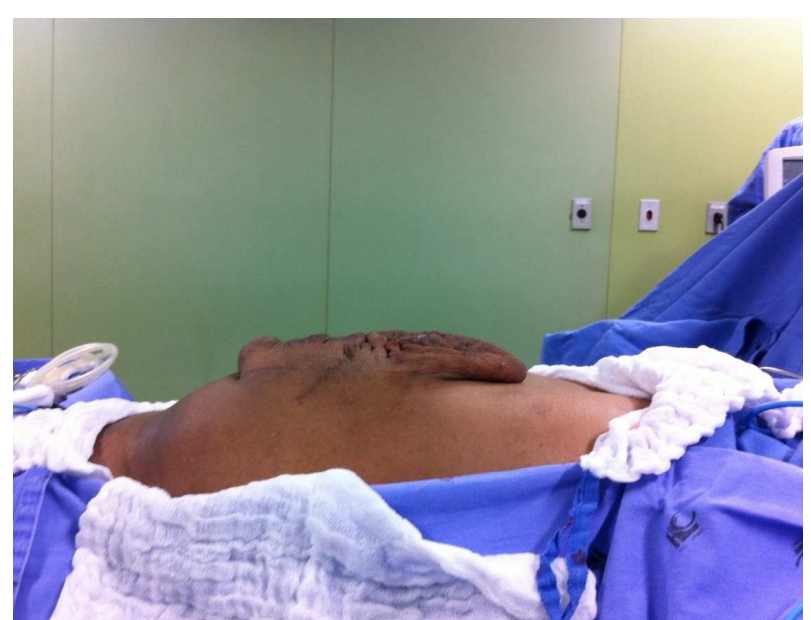

Fonte: Foto tirada no próprio serviço. 
FIGURAS 3 e 4 - Intra e pós operatório imediato

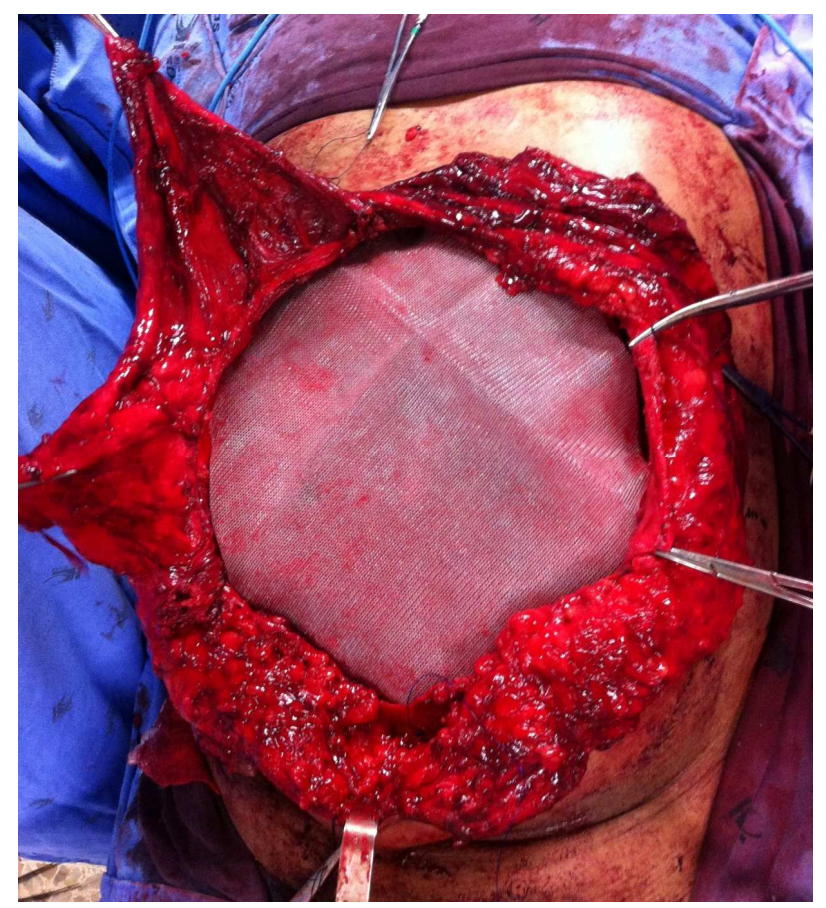

Fonte: Fotos tiradas no próprio serviço.

por 8 dias corridos, sempre seguido de enfaixamento abdominal.

No ato cirúrgico, realizado no dia 27/03/14, foi dissecado o espaço pre-peritonial e posicionada, primeiramente uma tela absorvivel (Gore Bio®) e, sobre esta, uma tela de polipropileno. Realizado fechamento por planos convencional com posicionamento de dreno PortoVac ${ }^{\circledR}$ no espaço supraaponeurótico e exérese do excesso de pele ocasionado pelo abaulamento crônico.

Evoluiu no pós operatório imediato sem intercorrências, em ambiente de terapia intensiva, sendo monitorizado a pressão intrabdominal por critérios clínicos, laboratoriais e pela medida seriada da pressão intravesical. Recebeu alta hospitalar no dia 05/04/2014, ainda com o dreno, mesmo drenando pouca quantidade de liquido serossanguinolento, posteriormente retirado na

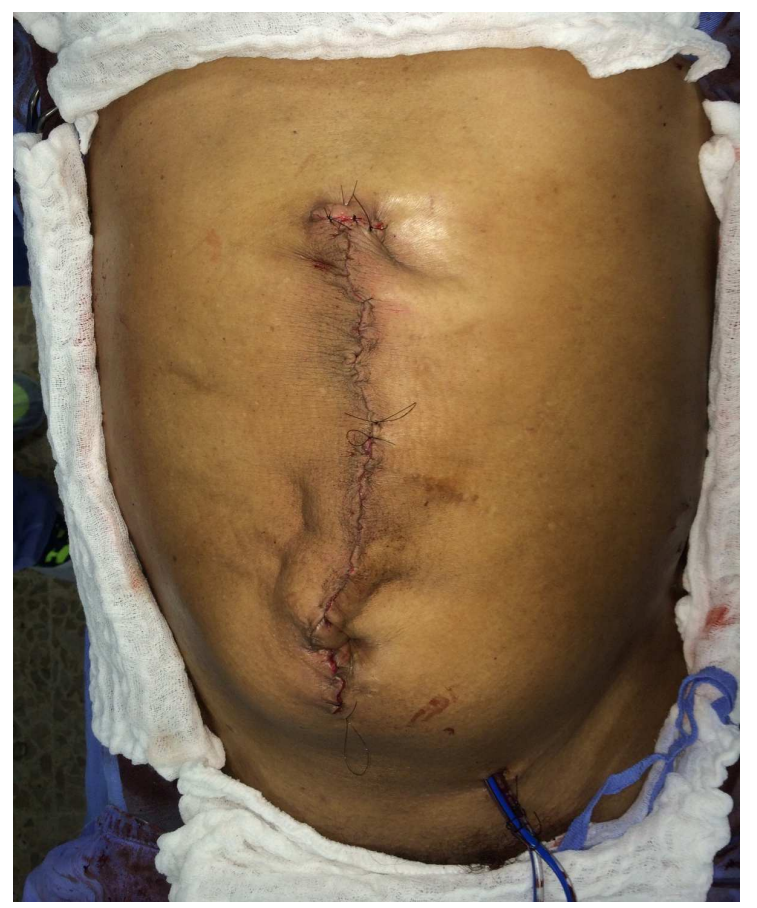

primeira consulta ambulatorial, 7 dias após a alta. Pós operatório remoto sem sinais de recidiva e apresentando boa qualidade de vida.

\section{DISCUSSÃO}

A redução do conteúdo herniário para a cavidade abdominal sem a presença da síndrome compartimental abdominal, caracterizada pela hipertensão abdominal associada a redução do retorno venoso, débito cardíaco, diurese e piora do padrão ventilatório no pós-operatório, reflete que o pneumoperitônio pré-operatório obteve sucesso (TANAKA, 2009; SPERANZINI, 2010). Existem porém poucas descrições na literatura acerca do uso do mesmo no preparo cirúrgico de hérnias incisionais gigantes, não havendo inclusive dados suficientes que surgiram o valor necessário de ar a ser insuflado na cavidade para que o pneumoperitônio seja 
efetivo (MASON, 2001; OLIVEIRA, 2003; MINOSSI, 2009). Em nosso trabalho realizamos insuflação total de $6420 \mathrm{~mL}$, valor considerado baixo comparado a outras publicações porém com efetividade, o que pode sugerir que o enfaixamento abdominal pós insuflação de ar ajuda a melhor distribuição do ar na cavidade levando a uma distensão mais homogênea, auxiliando no aumento do continente sem a necessidade de insuflação de grandes volumes, o que traz desconforto ao paciente e prolonga sua estadia hospitalar.

\section{CONCLUSÃO}

O presente trabalho demonstra a efetividade do pneumoperitônio pré-operatório em aumentar o continente para adequar-se ao conteúdo das hérnias incisionais gigantes com perda de domicílio e redução da incidência da síndrome compartimental. Sugerimos que mais trabalhos acerca do tema devem ser publicados para que se estabeleça um protocolo universal para realização do mesmo.

\section{REFERÊNCIAS}

GOÑI-MORENO I. Pneumoperitoneum. Surgery. 1947; 22: pp. 945

MASON, Edward E., Pneumoperitoneum in the treatment of giant hernias, with special reference to obesity. In: BENDAVID R, ABRAHAMSON J, ARREGUI M, FLAMENT JB, PHILIPS EH. Abdominal Wall Hernias. New york: Springer 2001 p. 675-679

MINOSSI, José Guilherme et al . O uso do pneumoperitônio progressivo no pré-operatório das hérnias volumosas da parede abdominal. Arq. Gastroenterol., São Paulo, v. 46, n. 2, June 2009 . 2015.

OLIVEIRA, Diogo Franco Vieira de et al . Pneumoperitôneo progressivo no préoperatório de hérnias incisionais volumosas. Rev. Col. Bras. Cir., Rio de Janeiro, v. 30, n. 4, Aug. 2003

\section{PINGUE JH, CLARCK JOHN H.}

Pneumoperitoneum A Neglected Procedure for the Repair of Large Abdominal Hernias, Arch Surg. 1968, 96(2):252-253

SPERANZINI, Manlio Basilio; DEUTSCH, Claudio Roberto. Grandes hérnias incisionais. ABCD, arq. bras. cir. dig., São Paulo, v. 23, n. 4, Dec. 2010.

TANAKA, Eduardo Yassushi. Análise de fatores preditivos de ressecção visceral no tratamento operatório de doentes portadores de hérnia incisional gigante com perda de domicílio submetidos a pneumoperitônio progressivo préoperatório. 2009. Tese (Doutorado em Clínica Cirúrgica) - Faculdade de Medicina, Universidade de São Paulo, São Paulo, 2009. 